\title{
Double-Frequency-Shift Acousto-Optic Modulator with Controllable Pulse Pair Frequency Difference
}

\author{
Yuhe Wang ${ }^{1,2}$, Yudong Lian ${ }^{1,2,3, *} \mathbb{C}$, Shiwei Han ${ }^{1,2}$, Yang Yu ${ }^{1,2}$, Xuan Qi ${ }^{1,2}$, Zhenxu Bai ${ }^{1,2,3}$, Yulei Wang ${ }^{1,2,3}$ \\ and Zhiwei Lu $1,2,3$ \\ 1 Center for Advanced Laser Technology, Hebei University of Technology, Tianjin 300401, China; \\ 201921902028@stu.hebut.edu.cn (Y.W.); 202021902024@stu.hebut.edu.cn (S.H.); \\ 202031903055@stu.hebut.edu.cn (Y.Y.); 202021902006@stu.hebut.edu.cn (X.Q.); zxbai@hebut.edu.cn (Z.B.); \\ wyl@hebut.edu.cn (Y.W.); zhiweilv@hebut.edu.cn (Z.L.) \\ 2 Hebei Key Laboratory of Advanced Laser Technology and Equipment, Tianjin 300401, China \\ 3 Tianjin Key Laboratory of Electronic Materials and Devices, Tianjin 300401, China \\ * Correspondence: ydlian@hebut.edu.cn
}

Citation: Wang, Y.; Lian, Y.; Han, S.; Yu, Y.; Qi, X.; Bai, Z.; Wang, Y.; Lu, Z. Double-Frequency-Shift Acousto-Optic Modulator with Controllable Pulse Pair Frequency Difference. Photonics 2021, 8, 436. https://doi.org/10.3390/ photonics 8100436

Received: 3 September 2021 Accepted: 30 September 2021 Published: 12 October 2021

Publisher's Note: MDPI stays neutral with regard to jurisdictional claims in published maps and institutional affiliations.

Copyright: (c) 2021 by the authors. Licensee MDPI, Basel, Switzerland. This article is an open access article distributed under the terms and conditions of the Creative Commons Attribution (CC BY) license (https:/ / creativecommons.org/licenses/by/ $4.0 /)$.

\begin{abstract}
A scheme for controlling the frequency difference of output pulse pair with double frequency shift loops is proposed. The frequency shift system includes two loop elements of 20 and $200 \mathrm{MHz}$. The first one carries out a single selective positive frequency shift of $1-20 \mathrm{MHz}$, and the second one can satisfy a single fixed positive frequency shift of $200 \mathrm{MHz}$. The reverse cascade technology of two acousto-optic crystals is introduced to solve the limitation of the small frequency shift of crystal size. A multichannel synchronization signal completes the time domain control of each acousto-optic modulator. Finally, the frequency shift difference of the output pulse pair ranges of $0-2 \mathrm{GHz}$, and the frequency shift accuracy is $5 \mathrm{MHz}$.
\end{abstract}

Keywords: acousto-optic modulation; Bragg diffraction; optical frequency shift; time synchronizer

\section{Introduction}

Since the discovery of light diffraction caused by ultrasound wave [1], researchers have made an in-depth exploration of acousto-optic interaction [2-5]. They used an acoustooptic effect to modulate the frequency, intensity and propagation direction of optical signal, and then design an acousto-optic tunable filter (AOTF) [6-8], acousto-optic deflector (AOD) [9-11], acousto-optic modulator (AOM) [12-14] and other optical devices, which are widely used in optical communication, laser control, spectrum detection and other fields [15-17]. Among them, an AOM uses diffraction to realize the frequency shift of the diffracted light relative to the incident light. In addition, the AOM has the function of an optical switch by adjusting the driving signal of the ultrasound field.

The effect of the internal acoustic field deforms the crystal and produces periodic density modulation. The final result is a periodic change in the refractive index of the crystal, which functions similarly to an "optical phase grating". When the light is injected into the crystal, the optical signal is affected by the "grating" and produces a diffraction effect. Acousto-optic diffraction can be divided into Raman-Nath Diffraction and Bragg Diffraction. As the multi-stage diffracted light in the process of Raman-Nath Diffraction will split the incident energy and result in large energy loss of primary diffraction light, it is not suitable for acousto-optic frequency shift modulation. In Bragg Diffraction, the output light only includes order 0 and \pm 1 (the positive and negative conditions depend on the incident direction), so that most of the incident energy is transferred to the diffracted light. Moreover, when the incident angle is the Bragg angle, nearly $100 \%$ diffraction efficiency can be obtained.

At present, most research on an acousto-optic frequency shift aims to obtain a broadband frequency shift above $\mathrm{GHz}$ with a high diffraction efficiency, which is usually realized 
by an all-optical fiber or integrated waveguide structure. However, there are relatively few studies on a single small frequency shift. In some fields, such as non-collinear stimulated Brillouin scattering (SBS) compression, a small frequency shift can improve the energy extraction efficiency of the system [18]. Moreover, heterodyne interference detection also needs a small frequency shift to improve the detection sensitivity [19]. At present, a small frequency shift has its particularity in some application scenarios, and related research is also in progress. Gazalet et al. [20] designed a frequency shifter with double $\mathrm{TeO}_{2}$ crystals and a large deflection angle, which can obtain a single frequency of $\pm 12 \mathrm{MHz}$ without changing the output angle of the frequency-shifted order. Kastelik et al. [21] loaded different RF signals into the same crystal to achieve the difference frequency output of diffraction light. Li et al. [22] completed the small-scale phase-modulation of incident light by using a series structure of two polarization-insensitive AOMs. If the frequencies of two RF drive signals are different, the structure can also achieve a small frequency shift of the output light. Dieulandard et al. [23] studied a tunable low-frequency optical frequency shifter, which is also based on the series connection of two acousto-optic crystals, and the final operation tuning range is extended to $42 \mathrm{MHz}$. Wang et al. [24] designed a low-frequency polarization maintaining fiber acousto-optic frequency shifter with a frequency shift of $10 \mathrm{MHz}$ and an insertion loss of $3.3 \mathrm{~dB}$. In general, double crystal cascade is an effective scheme to realize a small frequency shift. Using this method, researchers control the single frequency shift between 10-20 MHz.

Compared with previous reports, the double-frequency-shift loops in this design further reduces the amount of single frequency shift and improves the frequency shift accuracy. In addition, we integrate RF driver and acousto-optic modulators. By adjusting the synchronization signal, we can meet different frequency shift requirements, with high integration and strong operability. In this paper, a double-frequency-shift acoustooptic frequency modulator is designed. The frequency of the RF signal is controlled by a hardware circuit that further optimizes the cascade structure and controls the single frequency shift within $10 \mathrm{MHz}$. In addition, under the control of high-precision and low jitter synchronization signal, the maximum output frequency shift difference is $2 \mathrm{GHz}$, and the frequency shift accuracy reaches $5 \mathrm{MHz}$.

\section{System Structure}

The incident light of the system is a square pulse pair, which is obtained by modulating the single longitudinal mode continuous light through an arbitrary waveform generator and amplitude modulator. The central wavelength of two pulses is $1064 \mathrm{~nm}$ and they are both linearly polarized light. Oscilloscope waveform is shown in Figure 1.

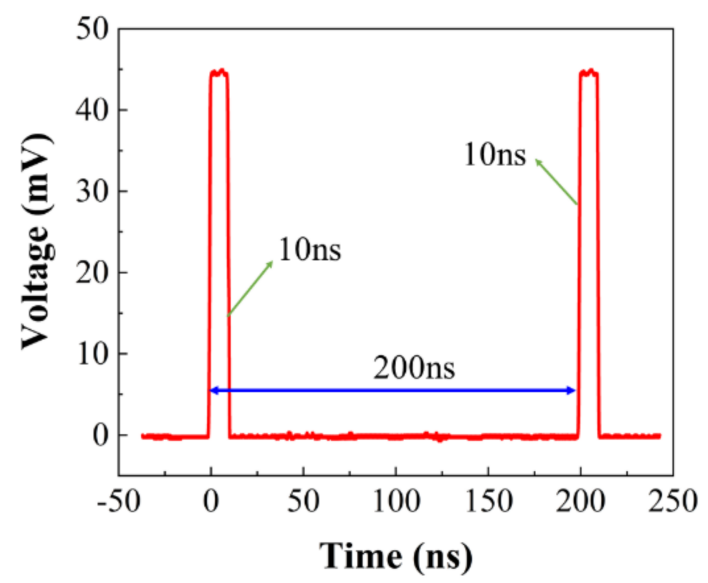

Figure 1. Time-domain waveform of Pulse pair source. 
In order to ensure that the acousto-optic device works in the Bragg diffraction region, the acousto-optic interaction length $L$ should meet the following conditions [24]:

$$
\begin{gathered}
L>2 L_{0} \\
L_{0}=n V^{2} / \lambda f^{2}
\end{gathered}
$$

where $L_{0}, n$ and $V$ represent the characteristic length, internal refractive index and sound velocity of the acousto-optic crystal, respectively; $\lambda$ is the working wavelength and $f$ stands for frequency shift. Equation (2) shows that the crystal length is inversely proportional to the square of the minimum frequency shift. It can be seen that if we want to realize the small frequency shift diffraction of an optical signal, we need to increase the size of the acousto-optic crystal, which is obviously not suitable for practical engineering applications. Double crystal reverse cascade technology, which is shown in Figure 2, follows the Bragg diffraction principle of single crystal.

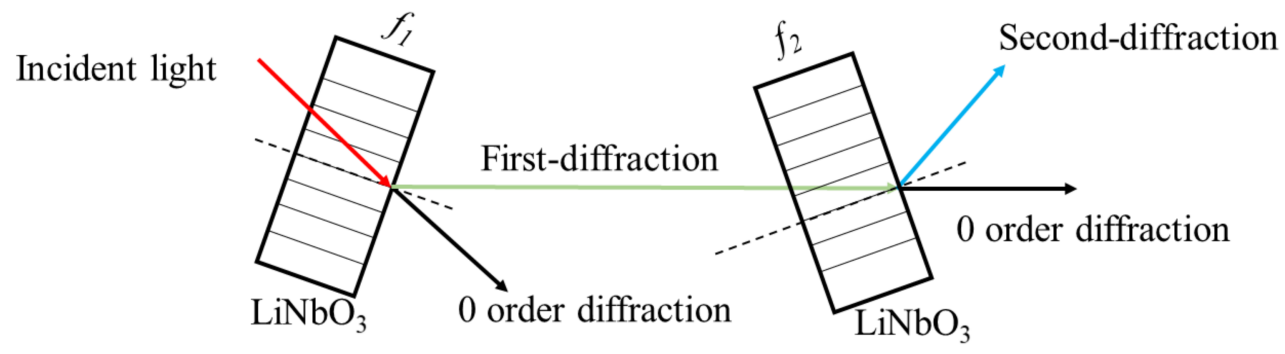

Figure 2. Working principle of double crystal cascade acousto-optic frequency shift.

The incident light enters the first acousto-optic crystal and interacts with the ultrasonic with frequency $f_{1}$ to produce the first diffraction. Its output is used as the incident light source of the second acousto-optic crystal, interacts with the ultrasonic with frequency $f_{2}$ to produce the second diffraction. Finally, the positive first-order diffraction of the second crystal is used as the output light of the device, as shown in Figure 2. At this time, the single minimum frequency shift is no longer limited by the crystal size, which is only related to the difference between the ultrasonic frequencies $f_{1}$ and $f_{2}$, and the frequency of the output light meets the following requirements:

$$
\Delta v=\left|f_{1}-f_{2}\right|
$$

The system introduces fiber coupling loops to perform multiple frequency shifts on the pulse continuously, and the frequency shift times and the selective output of the pulse sequence are controlled by multichannel time synchronization. The frequency shift experimental setup is shown in Figure 3. It consists of a single 1-20 MHz optional frequency shift loop (increment step is $1 \mathrm{MHz}$ ) and a single $200 \mathrm{MHz}$ fixed positive frequency shift loop. In order to ensure the orderly operation of the system, we provide six synchronization signals (CHX) for the system. These signals are input into the corresponding six acousto-optic modulators (AOMX) with different delays and widths. The delay of the synchronization signal determines the start time of the acousto-optic switch, and the width of the synchronization signal determines the duration of the acousto-optic switch.

Under the time domain division of AOM1 and AOM4, the incident pulse pair becomes two independent pulses $\left(\mathrm{P}_{1}\right.$ and $\left.\mathrm{P}_{2}\right)$ into the frequency shift loop. They enter 1-20 MHz-AOM and $200 \mathrm{MHz}-\mathrm{AOM}$ acousto-optic modulators, respectively, for an independent frequency shift. Through system test, the time for $P_{1}$ and $P_{2}$ to pass through the corresponding frequency shift loop is $100 \mathrm{~ns}$, and the total duration of the frequency shift $\Delta \mathrm{t}$ is determined by the synchronization signals $\mathrm{CH} 2$ and $\mathrm{CH} 5$. Since $\mathrm{P}_{1}$ and $\mathrm{P}_{2}$ immediately enter the acousto-optic crystal for a primary frequency shift when they just incident into the loop, the number of cycles of $P_{1}$ and $P_{2}$ is $(\Delta t / 100)-1$, and the number of frequency shifts is $\Delta t / 100$. Moreover, $2 \times 1 / 1 \times 2$ coupler is to form a closed loop in the frequency 
shift process, and its spectral ratio is 50:50. Multiple frequency shifts seriously affect the stability of the system; therefore, we limit the frequency shifts of pulses to 10 times, and a sequence of 10 pulses can be observed from the input of AOM3 and AOM6, which is shown in Figure 4. The delay and frequency difference between any two adjacent pulses of sequence are equal. In order to obtain the sub-pulse with an ideal frequency shift, it is necessary to use the acousto-optic switching function of AOM3 and AOM6 to filter the frequency shift components of the previous times under the control of synchronization signals $\mathrm{CH} 3$ and $\mathrm{CH} 6$.

(a)

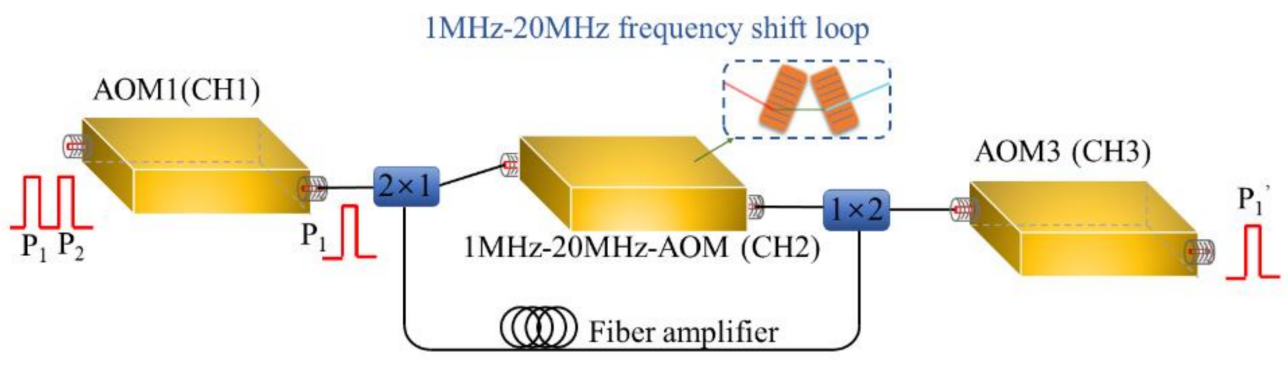

(b)

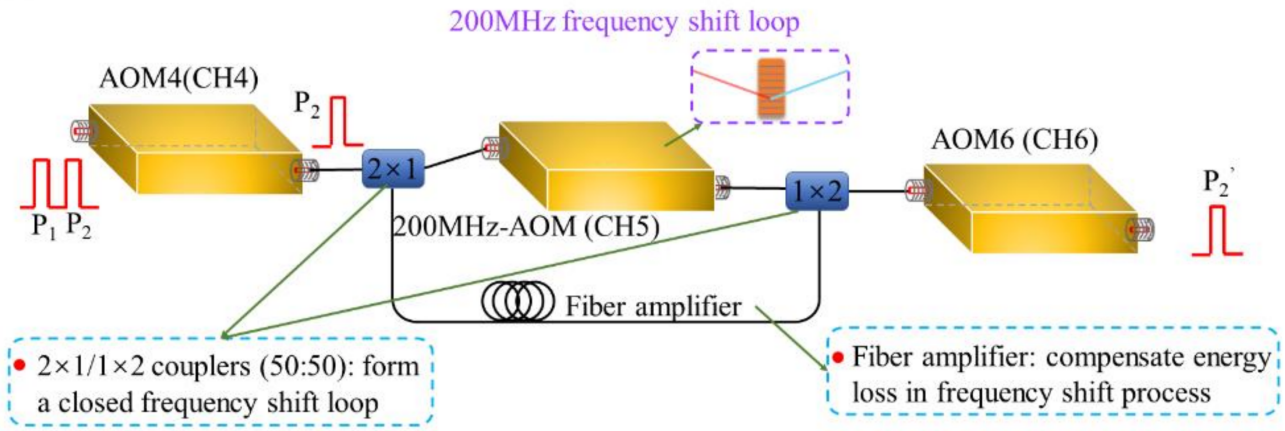

Figure 3. Optical path diagram of frequency shift loops: (a) 1-20MHz; (b) $200 \mathrm{MHz}$.

Ideal frequency shift output

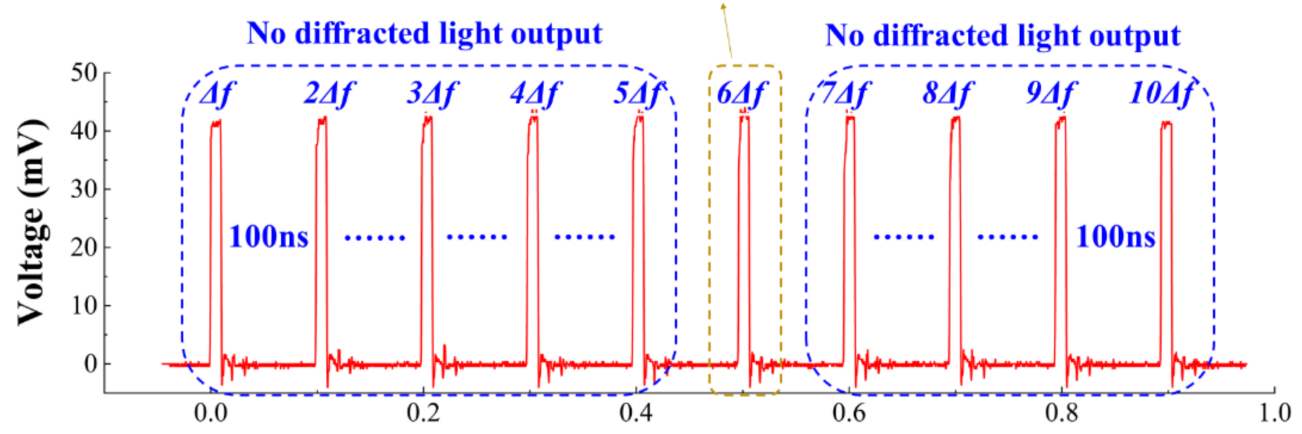

Time (us)

Figure 4. Schematic diagram of pulse sequence.

The frequency shift variation of the pulse after passing through the frequency shift loop is as follows:

$$
\left\{\begin{array}{c}
\Delta v_{1 M H z-20 M H z}=\Delta f \cdot m \\
\Delta v_{200 M H z}=200 \cdot n
\end{array}\right.
$$


where $\Delta f=1,2,3, \ldots, 20 \mathrm{MHz}$ is the selected single frequency shift, $m=n=1,2, \ldots, 10$ are the number of frequency shifts of $P_{1}$ and $P_{2}$, respectively. The frequency difference between the two pulses after beam combination is as follows:

$$
\Delta v^{\prime}=|\Delta f \cdot m-200 \cdot n|
$$

\section{Experiment Result}

The beat frequency method is used to measure the frequency difference between two pulses to test the effect of a frequency shift. The width of both pulses is $10 \mathrm{~ns}$. The frequency differences of $500 \mathrm{MHz}, 1 \mathrm{GHz}, 1.5 \mathrm{GHz}$ and $2 \mathrm{GHz}$ are tested, respectively, which are shown in Figure 5.
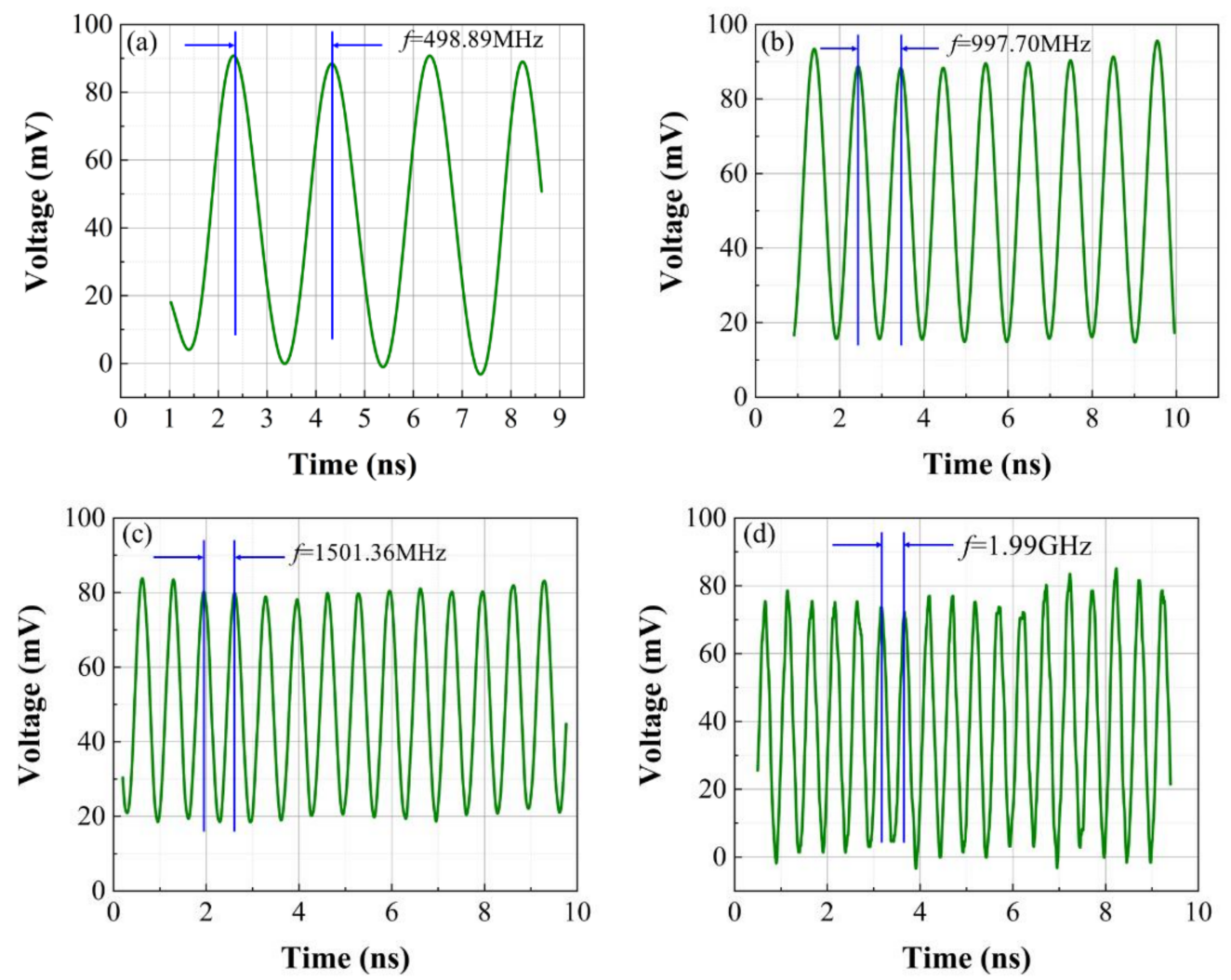

Figure 5. $500 \mathrm{MHz}$ interval beat frequency output: (a) $500 \mathrm{MHz}$; (b) $1 \mathrm{GHz}$; (c) $1.5 \mathrm{GHz}$; (d) $2 \mathrm{GHz}$.

The single increment of the above four frequency shifts is $500 \mathrm{MHz}$, and the errors with the ideal frequency shift are $0.22,0.23,0.09$ and $0.05 \%$, respectively. Finally, the double frequency shift loops acousto-optic modulation system realizes the maximum $2 \mathrm{GHz}$ frequency difference of two pulses. In fact, the minimum single frequency shift of the system is $1 \mathrm{MHz}$. Limited by the response time of the oscilloscope (Tektronix DSA71254C), photodetector (Thorlabs-DET08CFC/M) and the interference of the external environment, it is difficult to completely display the beat frequency result of a small frequency shift for one cycle, and the frequency shift accuracy of $1 \mathrm{MHz}$ cannot be observed using the beat frequency method. Therefore, the frequency shift increment accuracy of $5 \mathrm{MHz}$ is verified in this paper, and the results are shown in Figure 6. 


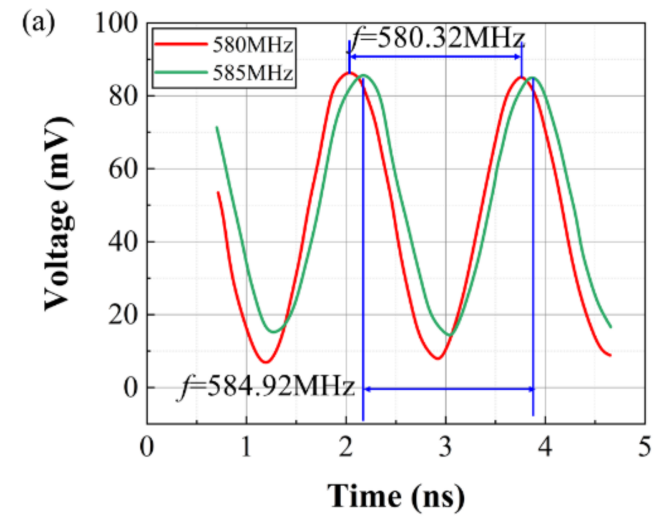

(a)

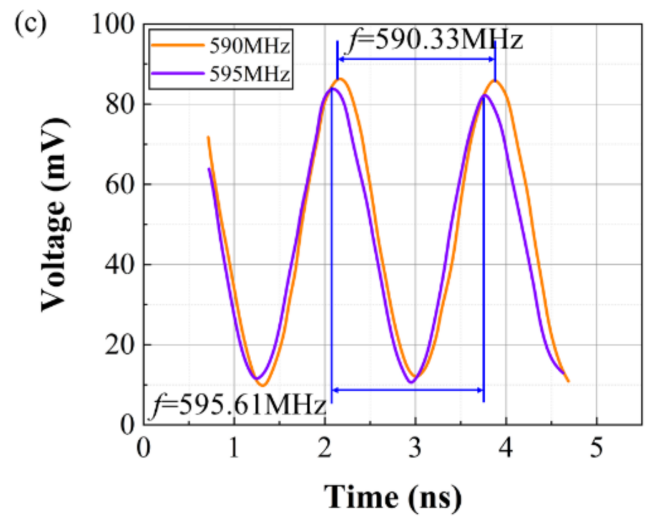

(c)

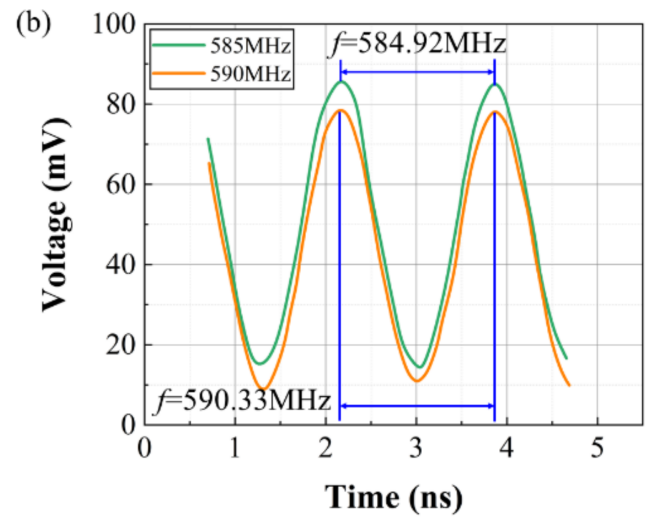

(b)

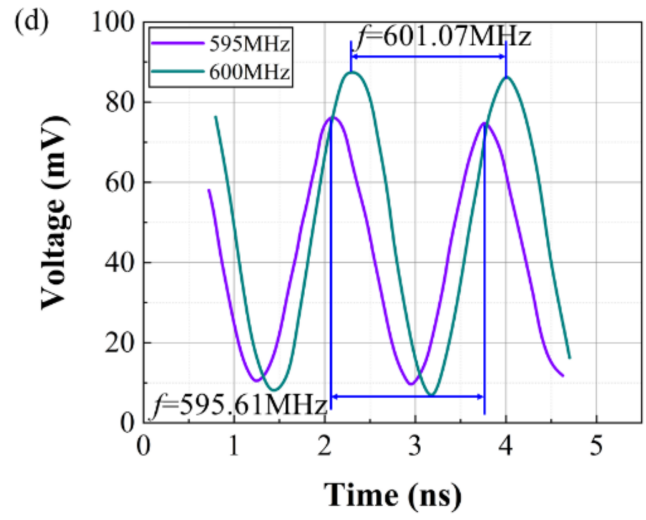

(d)

Figure 6. $5 \mathrm{MHz}$ interval beat frequency output: (a) 580-585 MHz; (b) 585-590 MHz; (c) 590-595 MHz; (d) 595-600 MHz.

It can be seen that the frequency difference intervals in the four cases are 4.6, 5.41, 5.28 and $5.46 \mathrm{MHz}$, respectively, and the average incremental error under the condition of a small frequency shift is $7.8 \%$. Although the accuracy of $1 \mathrm{MHz}$ is not tested, the single frequency shift of $5 \mathrm{MHz}$ is still innovative compared with the previous research on frequency shifter. Finally, we judge the frequency stability by testing the wavelength change of the system, as shown in Figure 7.

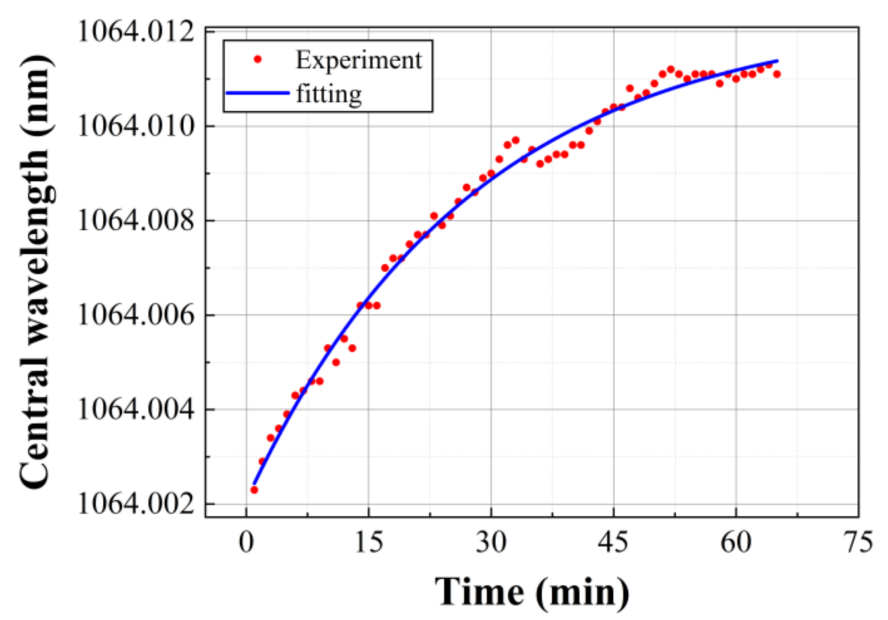

Figure 7. Schematic diagram of central wavelength drift.

It can be seen that the central wavelength has a small drift, which first increases rapidly and then tends to be flat. This is because the temperature will not fluctuate significantly 
after the system works for a period of time. The root mean square error (RMSE) of the central wavelength change is 0.0025 . This indicates that the system should work for more than half an hour before actual operation.

\section{Conclusion}

This paper introduces a double frequency shift loops acousto-optic modulator with a controllable output pulse frequency difference. In the design, double $\mathrm{LiNbO}_{3}$ crystal reverse cascade technology is used to solve the limitation of crystal size on a single small frequency shift. With the help of synchronous delay signal with high precision and low jitter, the orderly operation of the frequency shift loop is ensured. Finally, the maximum frequency shift difference of the system is $2 \mathrm{GHz}$, the single frequency shift accuracy is at least $5 \mathrm{MHz}$, and the frequency shift error is no more than $8 \%$. This special pulse light source can not only be used as Stokes seed light for SBS pulse compression, but also has application potential in the fields of optical fiber sensing, ultrasonic testing, etc.

Author Contributions: Conceptualization, Y.W. (Yuhe Wang) and Y.L.; data curation, S.H. and Y.Y.; formal analysis, Y.W. (Yuhe Wang); funding acquisition, Y.Y. and X.Q.; project administration, S.H.; supervision, Y.L., Z.B., Y.W. (Yulei Wang) and Z.L.; writing—original draft, Y.W. (Yuhe Wang); writing-review and editing, Y.W. (Yuhe Wang) and Y.L. All authors have read and agreed to the published version of the manuscript.

Funding: This research was funded by National Natural Science Foundation of China (Grant Nos 61905062, 61927815, 62075056 and 61905061); China Postdoctoral Science Foundation (Grant Nos 2020M670613); Hebei Postdoctoral Scholarship Project (Grant Nos B2020003026); Natural Science Foundation of Hebei Province (Grant Nos F2019202294) and Hebei Science and technology innovation strategy project (Grant Nos 20180601).

Institutional Review Board Statement: Not applicable.

Informed Consent Statement: Not applicable.

Data Availability Statement: Not applicable.

Conflicts of Interest: The authors declare no conflict of interest.

\section{References}

1. Raman, C.V.; Nagendra Nathe, N.S. The diffraction of light by high frequency sound waves: Part I. Proc. Indian Acad. Sci. 1935, 2, 406-412. [CrossRef]

2. Kim, B.Y.; Blake, J.N.; Engan, H.E.; Shaw, H.J. All-fiber acousto-optic frequency shifter. Opt. Lett. 1986, 11, 389-391. [CrossRef] [PubMed]

3. Kakio, S.; Zou, N.; Kitamura, M.; Ito, H.; Nakagawa, Y. An Integrated Acousto-optic Frequency Shifter Driven by Surface Acoustic Wave for 1.55 $\mu \mathrm{m}$ Optical Wavelength. Jpn. J. Appl. Phys 2003, 42, 30632003. [CrossRef]

4. Chou, C.C.; Lu, S.Y.; Lin, T.; Lu, S.H.; Jeng, R.J. Environment-noise-free optical heterodyne retardation measurement using a double-pass acousto-optic frequency shifter. Opt. Lett. 2016, 41, 5138-5141. [CrossRef]

5. Epikhin, V.M.; Baryshev, V.N.; Slyusarev, S.N.; Aprelev, A.V.; Blinov, I.Y. Acousto-optic modulators for a controlled frequency shift of light beams in optical and microwave cold-atom frequency standards. IEEE J. Quantum Electron. 2019, 49, 857-862. [CrossRef]

6. Gao, S.L. Sidelobe Problems of Integrated Optical Acousto-Optic Tunable Filter. Chin. J. Lasers 2019, 46, $182-197$.

7. Batshev, V.I.; Machikhin, A.S.; Kozlov, A.B.; Boritko, S.V.; Sharikova, M.O.; Karandin, A.V.; Pozha, V.E.; Lomonov, V.A. Tunable Acousto-Optic Filter for the 450-900 and 900-1700 nm Spectral Range. J. Commun. Technol. El+ 2020, 65, 800-805. [CrossRef]

8. Zhang, R.Y.; Li, P.L. Acousto-optic tunable flat top filter based on one-dimensional coupled-cavity photonic crystals. Acta Phys. Sin. 2021, 70, 054208.

9. Mazur, M.M.; Velikovskiy, D.Y.; Mazur, L.I.; Pavluk, A.A.; Pozhar, V.E.; Pustovoit, V.I. Elastic and photo-elastic characteristics of laser crystals potassium rare-earth tungstates $\mathrm{KRE}\left(\mathrm{WO}_{4}\right)_{2}$, where $\mathrm{RE}=\mathrm{Y}, \mathrm{Yb}, \mathrm{Gd}$ and Lu. Ultrasonics 2014, 54, 1311-1317. [CrossRef]

10. Kastelik, J.C.; Dupont, S.; Yushkov, K.B.; Molchanov, V.Y.; Gazalet, J. Double acousto-optic deflector system for increased scanning range of laser beams. Ultrasonics 2017, 80, 62-65. [CrossRef]

11. Shinada, H.; Sumi, K.; Shiina, T. Two-dimensional acousto-optic deflector using on-axis anisotropic Bragg diffraction for internal drum scanning exposure systems. Opt. Eng. 2017, 56, 085107. [CrossRef]

12. Shao, L.B.; Sinclair, N.; Leatham, J.; Hu, Y.W.; Yu, M.J.; Turpin, T.; Crowe, D.; Loncar, M. Integrated microwave acousto-optic frequency shifter on thin-film lithium niobate. Opt. Express 2020, 28, 23728-23738. [CrossRef] 
13. Wu, W.; Tang, S.; Wang, Z.L.; Linghu, A.M.; Zhu, J.; Fu, L.P. Single Polarization Fiber Acousto-Optic Modulator. Piezoelectr. Acoustoopt. 2020, 42, 37-39.

14. Pillai, G.; Li, S.S. Controllable multichannel acousto-optic modulator and frequency synthesizer enabled by nonlinear MEMS resonator. Sci. Rep. 2021, 11, 10898. [CrossRef]

15. Yushkov, K.B.; Molchanov, V.Y. Hyperspectral imaging acousto-optic system with spatial filtering for optical phase visualization. J. Biomed. Opt. 2017, 22, 66017. [CrossRef]

16. He, H.Y.; Sun, J.F.; Hou, P.P.; Zhang, B.; Xu, M.M.; Lao, C.Z. Angle Error Detection of Local Oscillator Nutation Based on Acousto-Optic Deflector in Fine Tracking System. Chin. J. Lasers 2018, 45, 218-224.

17. Machikhin, A.; Batshev, V.; Pozhar, V.; Naumov, A. Single-volume dual-channel acousto-optical tunable filter. Opt. Express 2020, 28, 1150-1157. [CrossRef]

18. Yuan, H.; Wang, Y.L.; Lu, Z.W.; Zheng, Z.X. Active frequency matching in stimulated Brillouin amplification for production of a $2.4 \mathrm{~J}, 200$ ps laser pulse. Opt. Lett. 2018, 43, 511-514. [CrossRef]

19. Liu, D.; Zheng, B.; Guo, H.L.; Liu, H.; Liu, N.Q. Micro-vibration measuring technology based on heterodyne interference. J. Appl. Opt. 2014, 35, 128-131.

20. Gazalet, M.G.; Ravez, M.; Haine, F.; Bruneel, C.; Bridoux, E. Acousto-optic low-frequency shifter. Appl. Opt. 1994, 33, 1293-1298. [CrossRef]

21. Kastelik, J.C.; Benaissa, H.; Dupont, S.; Pommeray, M. Acousto-optic tunable filter using double interaction for sidelobe reduction. Appl. Opt. 2009, 48, C4-C10. [CrossRef]

22. Li, E.B.; Yao, J.Q.; Yu, D.Y.; Xi, J.T.; Chicharo, J. Optical phase shifting with acousto-optic devices. Opt. Lett. 2005, 30, 189-191. [CrossRef] [PubMed]

23. Dieulangard, A.; Kastelik, J.C.; Dupont, S.; Gazalet, J. Acousto-optic wide band optical low-frequency shifter. Appl. Opt. 2013, 52, 8134-8141. [CrossRef] [PubMed]

24. Wang, X.X.; Chen, H.Z.; Wu, Z.C.; Wang, Z.L.; Zhou, D.; Liu, G.; Huo, Y.J. A polarization Maintaining Fiber Coupled AcoustoOptical Frequency Shifter Via Double Crystals For Low Frequency Shifting. Piezoelectr. Acoustoopt. 2016, 38, $394-397$. 